\title{
High phosphorus intake and gut-related parameters - results of a randomized placebo-controlled human intervention study
}

Ulrike Trautvetter $^{1 *}$ (D) Amélia Camarinha-Silva ${ }^{2}$, Gerhard Jahreis ${ }^{3,5}$, Stefan Lorkowski ${ }^{4,5}$ and Michael Glei ${ }^{1,5}$

\begin{abstract}
Background: In recent years, high phosphate intakes were discussed critically. In the small intestine, a part of the ingested phosphate and calcium precipitates to amorphous calcium phosphate (ACP), which in turn can precipitate other intestinal substances, thus leading to a beneficial modulation of the intestinal environment. Therefore, we analysed faecal samples obtained from a human intervention study regarding gut-related parameters.
\end{abstract}

Methods: Sixty-two healthy subjects (men, $n=30$; women, $n=32$ ) completed the double-blind, placebo-controlled and parallel designed study (mean age: $29 \pm 7$ years; mean BMl: $24 \pm 3 \mathrm{~kg} / \mathrm{m}^{2}$ ). Supplements were monosodium phosphate and calcium carbonate. During the first 2 weeks, all groups consumed a placebo sherbet powder, and afterwards a sherbet powder for 8 weeks according to the intervention group: P1000/Ca0 (1000 mg/d phosphorus), P1000/Ca500 (1000 mg/d phosphorus and $500 \mathrm{mg} / \mathrm{d}$ calcium) and P1000/Ca1000 (1000 mg/d phosphorus and $1000 \mathrm{mg} / \mathrm{d}$ calcium). After the placebo period and after 8 weeks of intervention faecal collections took place. We determined in faeces: short-chain fatty acids (SCFA) and fat as well as the composition of the microbiome (subgroup) and cyto- and genotoxicity of faecal water (FW). By questionnaire evaluation we examined tolerability of the used phosphorus supplement.

Results: Faecal fat concentrations did not change significantly due to the interventions. Concentrations of faecal total SCFA and acetate were significantly higher after 8 weeks of P1000/Ca500 supplementation compared to the P1000/Ca0 supplementation. In men, faecal total SCFA and acetate concentrations were significantly higher after 8 weeks in the P1000/Ca1000 group compared to the P1000/Ca0 one. None of the interventions markedly affected cyto- and genotoxic activity of FW. Men of the P1000/Ca1000 intervention had a significantly different gut microbial community compared to the men of the P1000/Ca0 and P1000/Ca500 ones. The genus Clostridium XVIII was significantly more abundant in men of the P1000/Ca1000 intervention group compared to the other groups. Supplementations did not cause increased intestinal distress.

Conclusions: The used high phosphorus diet did not influence cyto- and genotoxicity of FW and the concentrations of faecal fat independent of calcium intake. Our study provides first hints for a potential phosphorus-induced modulation of the gut community and the faecal total SCFA content.

Trial registration: The trial is registered at ClinicalTrials.gov as NCT02095392.

Keywords: Phosphorus intake, Phosphate intake, Calcium intake, Human study, Faecal water, Cytotoxicity, Genotoxicity, Short-chain fatty acids

\footnotetext{
* Correspondence: Ulrike.Trautvetter@uni-jena.de

'Department of Nutritional Toxicology, Institute of Nutrition, Friedrich Schiller

University Jena, Dornburger Straße 24, 07743 Jena, Germany

Full list of author information is available at the end of the article
} 


\section{Background}

Dietary phosphate and serum phosphate concentrations of healthy people and patients with chronic kidney disease have been discussed critically in the last years, regarding bone, cardiovascular health and mortality [1-4]. Whereas associations of phosphate concentrations in serum with cardiovascular diseases and mortality have been well established [2], it is still unclear whether dietary phosphorus influences serum phosphate concentrations [5, 6].

According to the National Health and Nutrition Examination Survey of the USA, dietary phosphorus intake of Americans exceeds the daily recommended intake of $700 \mathrm{mg}$ phosphorus for adults and the elderly [4, 7]. This is mainly a result of the increased consumption of food prepared or treated with phosphate additives, which ranged from baked goods to cola beverages [8, 9]. In 2014, our department determined the influence of a high phosphorus intake in combination with different calcium supplies on phosphorus, calcium, magnesium and iron metabolism as well as fibroblast growth factor 23 in a human intervention study [10]. In this and in other intervention studies, increased concentrations of phosphorus and calcium in the faeces after high calcium and phosphorus supplementation compared to placebo were found [10-13]. However, up to now, only a few studies dealt with the question, which effects are provoked by higher intestinal concentrations of calcium and phosphorus in the human gut. It is known that calcium and phosphate form amorphous calcium phosphate complexes $(\mathrm{ACP})$ in the small intestine. Intestinal substances and food ingredients, such as bile and fatty acids, can bind to ACP $[13,14]$. This leads to several physiological changes in the human gut, e.g. a modulation of the composition of faecal bile acids, faecal short-chain fatty acids (SCFA) and gut microbiota [11, 13]. Furthermore, high phosphorus intakes can lead to disturbances in the gut, like diarrhoea [15].

In the study presented here, we analysed faecal samples from the subjects of the above-mentioned study [10] regarding cytotoxicity and genotoxicity of faecal water (FW), faecal concentrations of SCFA and fat as well as the composition of the gut microbiome (in a subgroup of study subjects). In addition, we examined the tolerability of the used phosphorus supplement with respect to gut distress, such as diarrhoea or stomach ache, by questionnaire evaluation.

\section{Methods}

\section{Supplements}

In the current study, two supplements were used: monosodium phosphate $\left(\mathrm{NaH}_{2} \mathrm{PO}_{4} ; \mathrm{cfb}\right.$, Budenheim, Germany) and calcium carbonate $\left(\mathrm{CaCO}_{3}\right.$; $\mathrm{cfb}$, Budenheim, Germany). In order to achieve a supplementation of additional $1000 \mathrm{mg}$ phosphorus/d as well as additional 500 or $1000 \mathrm{mg}$ calcium/d, monosodium phosphate and calcium carbonate were mixed with sherbet powder. Sherbet powder without additional supplements served as placebo. Participants were encouraged to drink the sherbet powder twice a day diluted in $250 \mathrm{ml}$ water and to document when the sherbet powder was not consumed. Detailed information has been published elsewhere [10].

\section{Subjects and study design}

The study was conducted by the Institute of Nutrition of the Friedrich Schiller University Jena between March and July 2014.

Sixty-six omnivorous healthy subjects (men, $n=33$; women $n=33$ ) participated in this double-blinded, placebo-controlled and parallel designed study. Eligibility criteria for participants were an age between 18 and 60 years as well as mental and physical well-being. Exclusion criteria were regular intake of dietary supplements, renal diseases, pregnancy, nursing as well as post-menopausal age. Renal diseases were determined with the Chronic Kidney Disease Epidemiology Collaboration equation for estimating the glomerular filtration rate [16]. Subjects with baseline glomerular filtration rates of $<80 \mathrm{ml} / \mathrm{min} / 1.73 \mathrm{~m}^{2}$ were excluded.

The volunteers were provided with detailed information regarding purpose, course, and possible risks of the study. This study was conducted according to the guidelines laid down in the Declaration of Helsinki and all procedures involving human subjects were approved by the Ethical Committee of the Friedrich Schiller University Jena (no. 3987-01/14). Written informed consent was obtained from all subjects. The trial is registered at ClinicalTrials.gov as NCT02095392. Four participants dropped out because of illness and personal reasons. The remaining 62 volunteers (men, $n=30$; women, $n=32$ ) aged $29 \pm 7$ years and had a mean BMI of $24 \pm 3 \mathrm{~kg} / \mathrm{m}^{2}$.

The details of the study design have been published previously [10]. In short, 62 healthy subjects completed a double-blinded, placebo-controlled and parallel designed study. During the first two weeks, all groups consumed a placebo sherbet powder and afterwards, for eight weeks, a supplemented sherbet powder according to the intervention group: P1000/Ca0 (1000 mg/d phosphorus), P1000/ Ca500 (1000 mg/d phosphorus and $500 \mathrm{mg} / \mathrm{d}$ calcium) and P1000/Ca1000 (1000 mg/d phosphorus and $1000 \mathrm{mg} /$ $\mathrm{d}$ calcium). Dietary records, fasting blood samplings and urine collections took place (after placebo, four and eight weeks of supplementation). After two weeks of placebo and eight weeks of intervention subjects collected one faeces sample. Figure 1 shows the flow chart with the number of subjects included and excluded on each analysis. 


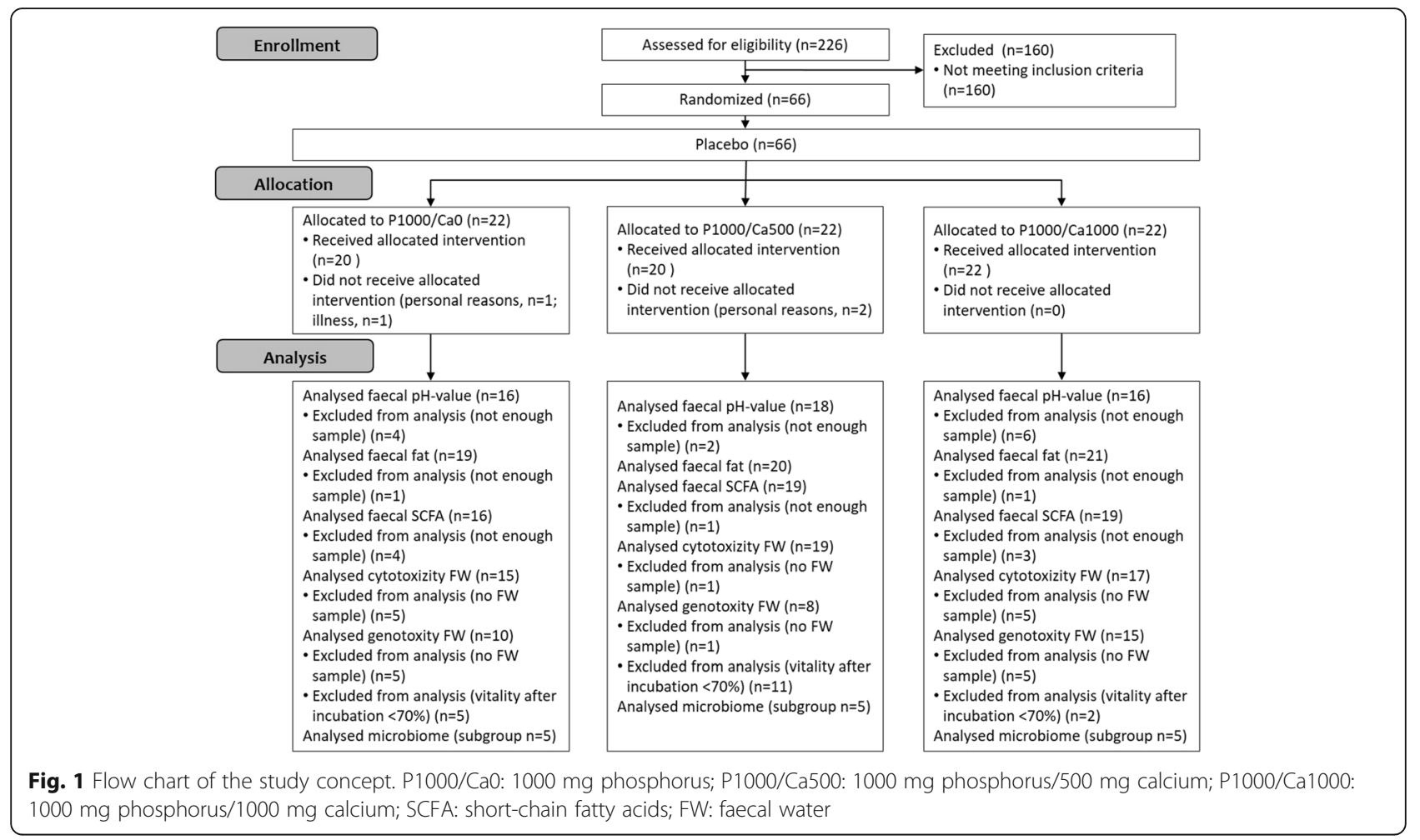

\section{Supplement tolerance questionnaire}

After placebo as well as after four and eight weeks of supplementation, subjects were encouraged to fill out a questionnaire about diverse health aspects for the last weeks. One question aimed to assess complaints regarding gut health. The subjects should report, whether they had problems with diarrhoea, obstipation, flatulence or undefined stomach ache. In the case of such problems, the subjects were asked to state, whether these problems could be due to the consumption of the test products (supplemented or non-supplemented powder).

\section{Faecal analysis}

At the evening before or at the morning of the blood sampling, the subjects were encouraged to collect one whole defecation in provided boxes and to store the sample in a cool dark place. The faecal samples were transported to the study centre at the day of the blood sampling after placebo and eight weeks of intervention. Each specimen was weighed and homogenised. Faecal $\mathrm{pH}$ value was measured using a glass pH electrode (InLab 420 electrode MP 225, Mettler Toledo GmbH, Giessen, Germany). Faeces samples were aliquoted for the respective analysis (faecal fat and FW preparation) and stored at $-20{ }^{\circ} \mathrm{C}$ until use. For SCFA analysis, $1 \mathrm{~g}$ fresh faeces were diluted with $2 \mathrm{ml}$ distilled water, mixed and stored at $-20{ }^{\circ} \mathrm{C}$ until use.

\section{Faecal fat}

Faecal fat was measured as ether extract after acid hydrolysis by conventional Soxhlet Extraction on a SOXTHERM 2000 automatic (C. Gerhardt, Königswinter, Germany) [13].

\section{Short-chain fatty acids in faeces}

SCFA analysis was performed as published by [17]. Briefly, faeces-water mixtures were thawed and thoroughly mixed. After centrifugation $(6000 \mathrm{x} \mathrm{g}$, $15 \mathrm{~min}$ ), $500 \mu \mathrm{l}$ of the supernatant were added with $50 \mu \mathrm{l} i$-caproic acid (internal standard), mixed and centrifuged $(6000 \mathrm{x} \mathrm{g}, 15 \mathrm{~min})$. For gas chromatographic measurements, $1 \mu \mathrm{l}$ of the solution was used (Shimadzu model GC 17A, Shimadzu, Kyoto, Japan).

\section{Faecal water preparation}

Fresh faeces samples were homogenised and diluted 1:1 with phosphate buffered saline (PBS) according to Klinder et al. [18]. Afterwards the faeces-PBS mixture was centrifuged for $4 \mathrm{~h}$ at $51500 \mathrm{x} \mathrm{g}$ at $4{ }^{\circ} \mathrm{C}$. The supernatant (FW) was aliquoted and stored at $-80{ }^{\circ} \mathrm{C}$.

\section{Cell culture and cytotoxicity of faecal water}

In the present analysis, the human colorectal adenocarcinoma cell line HT29 (American Type Culture Collection no. HTB-38, Rockville, MD, USA) was used for in vitro experiments. The specific properties and cell culture conditions have been described previously [19]. 
Briefly, cells were grown in Dulbecco's modified eagle medium (DMEM, Biochrom, Berlin, Germany), which was supplemented with $10 \%(v / v)$ fetal bovine serum (FBS Superior, Biochrom, Berlin, Germany) and 1\% (v/v) antibiotics (penicillin/streptomycin, Biochrom, Berlin, Germany). Within about $24 \mathrm{~h}$ the cells doubled their number under given laboratory conditions. In the experiments, passages 10-15 were used. To exclude contamination with mycoplasma, a mycoplasma test (MycoAlert Detection Kit, Lonza, Cologne, Germany) was performed [20].

To determine the cellular vitality after FW incubation, the trypan blue exclusion test, which is based on changes in membrane integrity of the treated cells, was used [21]. For this, the harvested HT29 cell suspension was incubated with trypan blue (Trypan Blue Solution, Sigma Aldrich, Steinheim, Germany) in a 1:1 dilution, followed by automatically counting of viable and dead cells using the ViCell XR (Beckman Coulter, Brea, CA, USA).

\section{Genotoxicity of faecal water}

Genotoxicity of FW was tested in HT29 cells as proposed by Oberreuther-Moschner et al. [22]. Briefly, cells were harvested and incubated with FW for $30 \mathrm{~min}$ at $37{ }^{\circ} \mathrm{C}$. Single cell microgel electrophoresis (comet assay) was used to analyse genotoxic activity of FW. For this, cells were embedded into agarose on microscopical slides, lysed and subjected to electrophoresis [17, 23]. Each FW sample was analysed in triplicates. PBS as negative control and $75 \mu \mathrm{M} \mathrm{H}_{2} \mathrm{O}_{2}$ as positive control were included for each assay, in order to ensure the performance of the comet assay. Microscopic evaluation was performed using the Comet Assay IV image analysis system (Perceptive Instruments, Suffolk, UK). The extent of DNA migration was determined for 60 spots per slide. The tail intensity (TI) was used as the marker for evaluation. The TI is defined as the proportion of tail fluorescence intensity of total comet fluorescence intensity.

\section{DNA extraction and sequencing}

A total of 30 faecal samples (five of each intervention group, thereof three men and two women) were extracted with PowerSoil $^{\circ}$ DNA Isolation Kit (MO BIO, Carlsbad, CA, USA) following the manufacturer's protocol. DNA was quantified using a NanoDrop 2000 spectrophotometer (Thermo Scientific, Waltham, MA, USA) and stored at $-20{ }^{\circ} \mathrm{C}$ until further analysis. Illumina library preparation was performed according to Camarinha-Silva et al. [24] with a slightly modified sequence of the primer 27F (AGRGTTHGATYMTGGCTCAG). Amplicons were verified by agarose gel electrophoresis, purified and normalized using SequalPrep Normalization Kit (Invitrogen, Carlsbad, CA,
USA). Samples were sequenced using $250 \mathrm{bp}$ paired-end sequencing chemistry on an Illumina MiSeq platform. Raw reads were quality filtered, assembled and aligned using the Mothur pipeline [25]. A total of 36,915 \pm 1233 sequencing reads were obtained per sample. The UCHIME algorithm was used to find possible chimeras, and reads were clustered at $97 \%$ identity into 832 operative taxonomic units (OTU). Only OTUs present on average abundance higher than $0.002 \%$ and a sequence length $>250 \mathrm{bp}$ were considered for further analysis. The closest representative was manually identified with seqmatch from the Ribosomal Database Project classifier [26]. Sequences were submitted to the European Nucleotide Archive under the accession number PRJEB2203.

\section{Statistics}

Data analysis was performed using the SPSS Statistics 21 statistical software package (IBM, Chicago, IL, USA). Power calculation for the primary outcome (plasma phosphate concentration) of the human intervention study was published elsewhere [10]. Variance homogeneity and normal distribution were tested using the Levene and the Kolmogorov-Smirnov test, respectively. The effect of supplementation between groups was tested using univariate analysis of variance followed by Bonferroni post-hoc test. Effects of time and gender were tested with paired and unpaired Students t-test, respectively. Differences were considered significant at $p \leq 0.05$. Values in the text and tables are means with standard deviations.

The amplicon sequencing data set was standardised by total, and the community similarity structure was depicted through non-metric multidimensional scaling plots and Principal Coordinate Analysis by calculating the Bray-Curtis similarity matrix using PRIMER version 7.0.9 (PRIMER-E, Plymouth Marine Laboratory, Plymouth, UK) [27]. PERMANOVA routine, based on the pair-wise tests using a permutation method under a reduced model, was used to study the significant differences and interactions between factors (diet, time point and gender). Similarity percentages analysis (SIMPER) identified the species contribution to the Bray-Curtis similarity among samples within each diet [27]. Differences in the abundance of OTUs of interest between diets were evaluated using the unpaired Welch's t-test that can handle unequal variances, unequal sample sizes and non-parametric data [28]. OTU abundances were considered significantly different for $p<0.05$.

\section{Results}

Baseline characteristics of subjects who completed the study and nutrient intake (three-day dietary record) have been published elsewhere [10]. Briefly, age, BMI, serum 25-hydroxyvitamin D, kidney function as well as intake 
of fat, protein and carbohydrates were not significantly different between the three study groups. Phosphorus and calcium intake increased significantly after the respective supplementations.

\section{Supplement tolerance questionnaire}

After consumption of placebo and the test products, 20$40 \%$ of subjects in each intervention group and at each time point reported complaints with gut health in the last four weeks (Fig. 2a). The majority of these subjects established a subjectively connection to the sherbet powder. This was independent of the respective phosphorus/ calcium supplementation or placebo (Fig. 2b). The main complaints according to the questionnaire were diarrhoea and flatulence; a few subjects also reported obstipation and undefined stomach ache (Fig. 2c).

\section{Faecal $\mathrm{pH}$ value and fat concentration}

After P1000/Ca0 and P1000/Ca500 intervention, the faecal $\mathrm{pH}$ value did not change (Table 1). In the whole study collective and in male subjects, the $\mathrm{pH}$ value decreased significantly due to P1000/Ca1000 intervention after eight weeks compared to placebo (Table 1). Noteworthy, this effect was likely caused by one male subject with a faecal $\mathrm{pH}$ value of 9.1 after placebo.

Faecal fat concentrations did not change significantly due to the interventions (Table 1).

\section{Concentration of faecal SCFA}

Considering men and women together, the interventions did not affect the concentrations of total SCFA or the concentrations of the main SCFA acetate, propionate and butyrate compared to placebo (Fig. 3). Certainly, concentrations of total SCFA and acetate were significantly higher after eight weeks of $\mathrm{P} 1000 / \mathrm{Ca} 500$ supplementation compared to the $\mathrm{P} 1000 / \mathrm{Ca} 0$ (Fig. 3). A gender-specific analysis revealed that acetate and total SCFA concentrations were significantly higher in the $\mathrm{P} 1000 / \mathrm{Ca} 1000$ group compared to the $\mathrm{P} 1000 / \mathrm{Ca} 0$ group after eight weeks, but only in male subjects (Additional file 1: Figure S1). The faecal concentrations of SCFA in the female subjects did not change (Additional file 2: Figure S2).

\section{Cytotoxicity and genotoxicity of faecal water}

After incubation with FW, vitality of the HT29 cells ranged on average between 73 and $83 \%$. For the P1000/Ca1000 intervention group, incubation of HT29 cells with FW from the eight-week time-point resulted in a significantly higher vitality of the cells compared to placebo (Fig. 4a). The interventions with phosphorus did not significantly affect the TI as a marker for genotoxic activity of the FW (Fig. 4b), considering men and women together and separately. Certainly, FW obtained from $\mathrm{P} 1000 / \mathrm{Ca} 0$ subjects

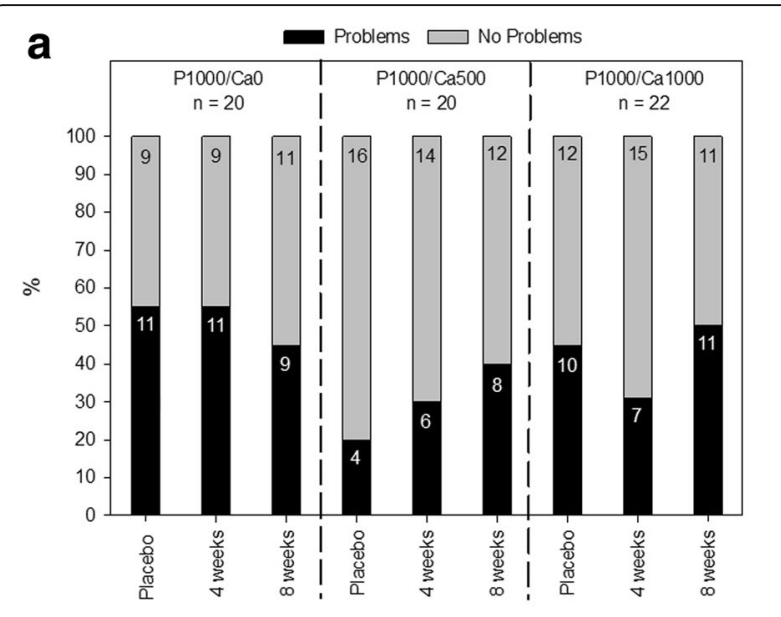

b

b Association to testproduct $\square$ No association to testprodukt
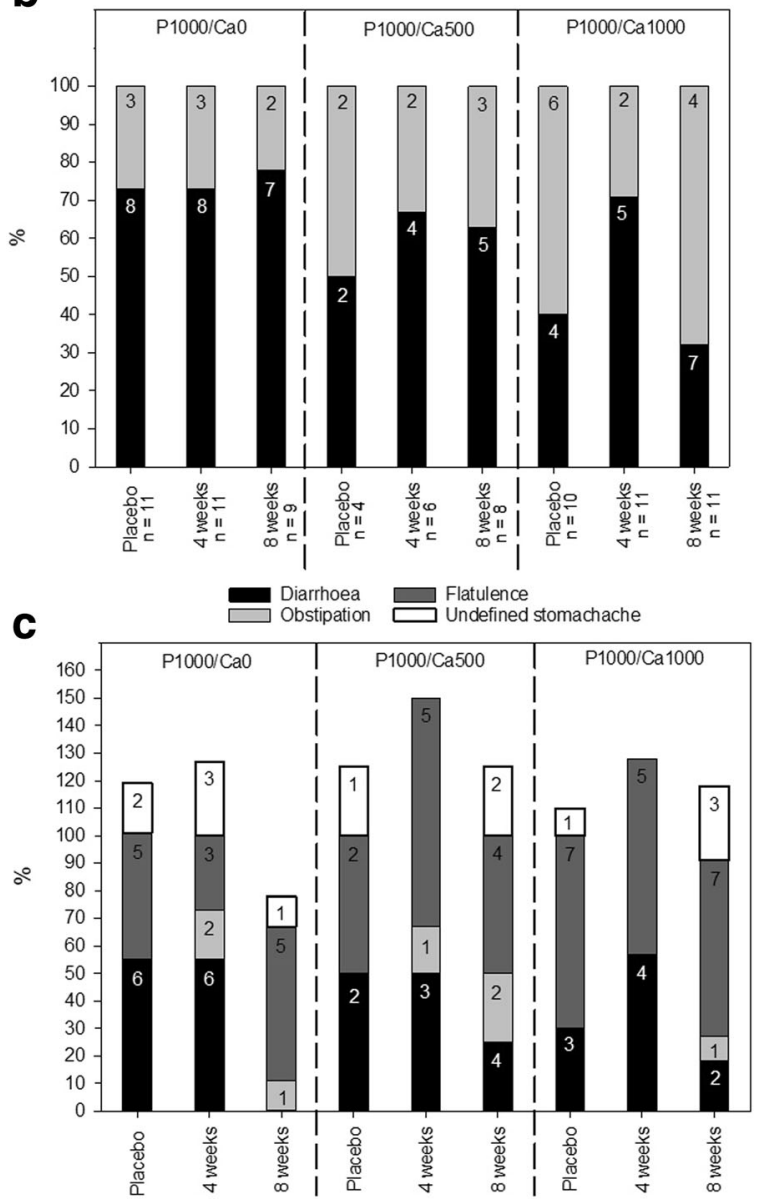

Fig. 2 Overview of intestinal disturbances after supplementation with phosphorus and calcium. a: Proportional (y-axis) and absolute (numbers in the bars) distribution of subjects reporting problems with gut health or not; b: proportional (y-axis) and absolute (numbers in the bars) distribution of reported associations with the test product, when subjects reported complaints; c proportional (yaxis) and absolute (numbers in the bars) distribution of types of reported problems; P1000/Ca0: 1000 mg phosphorus/0 mg calcium; P1000/Ca500: 1000 mg phosphorus/500 mg calcium; P1000/Ca1000: $1000 \mathrm{mg}$ phosphorus/1000 mg calcium 
Table 1 Faecal pH values and faecal fat concentrations after intervention with phosphorus and calcium

\begin{tabular}{ccll}
\hline Parameter & P1000/Ca0 & P1000/Ca500 & P1000/Ca1000 \\
\hline Faecal pH value & & & \\
$\mathrm{n}$ & 16 & 18 & 16 \\
Placebo & $6.7 \pm 0.7$ & $6.6 \pm 0.5$ & $7.0 \pm 0.7$ \\
8 weeks & $6.5 \pm 0.5$ & $6.3 \pm 0.5$ & $6.5^{*} \pm 0.6$ \\
Faecal fat [g/100 g fresh faeces] & & \\
n & 19 & 20 & 21 \\
Placebo & $5.9 \pm 2.8$ & $5.6 \pm 3.2$ & $4.1 \pm 1.7$ \\
8 weeks & $5.5 \pm 3.1$ & $5.6 \pm 2.5$ & $4.0 \pm 2.3$
\end{tabular}

Data are expressed as mean \pm standard deviation

P1000/Ca0 $1000 \mathrm{mg}$ phosphorus/0 mg calcium, P1000/Ca500 $1000 \mathrm{mg}$ phosphorus/500 mg calcium, P1000/Ca1000 $1000 \mathrm{mg}$ phosphorus $/ 1000 \mathrm{mg}$ calcium

significantly different to placebo $(p \leq 0.05)$; effect of time was tested with paired Students t-test

showed significantly higher genotoxic activity before the intervention compared to those from the P1000/ Ca1000 group (Fig. 4b). Based on our results that PBS (negative control) caused no genotoxic damages (TI $7 \pm 2 \%$ ) and $\mathrm{H}_{2} \mathrm{O}_{2}$ caused strong genotoxic effects (TI $59 \pm 15 \%$ ), the FW matrix showed only moderate genotoxicity independent of the phosphorus and calcium interventions (mean TI for all subjects $25 \pm$ $13 \%)$.

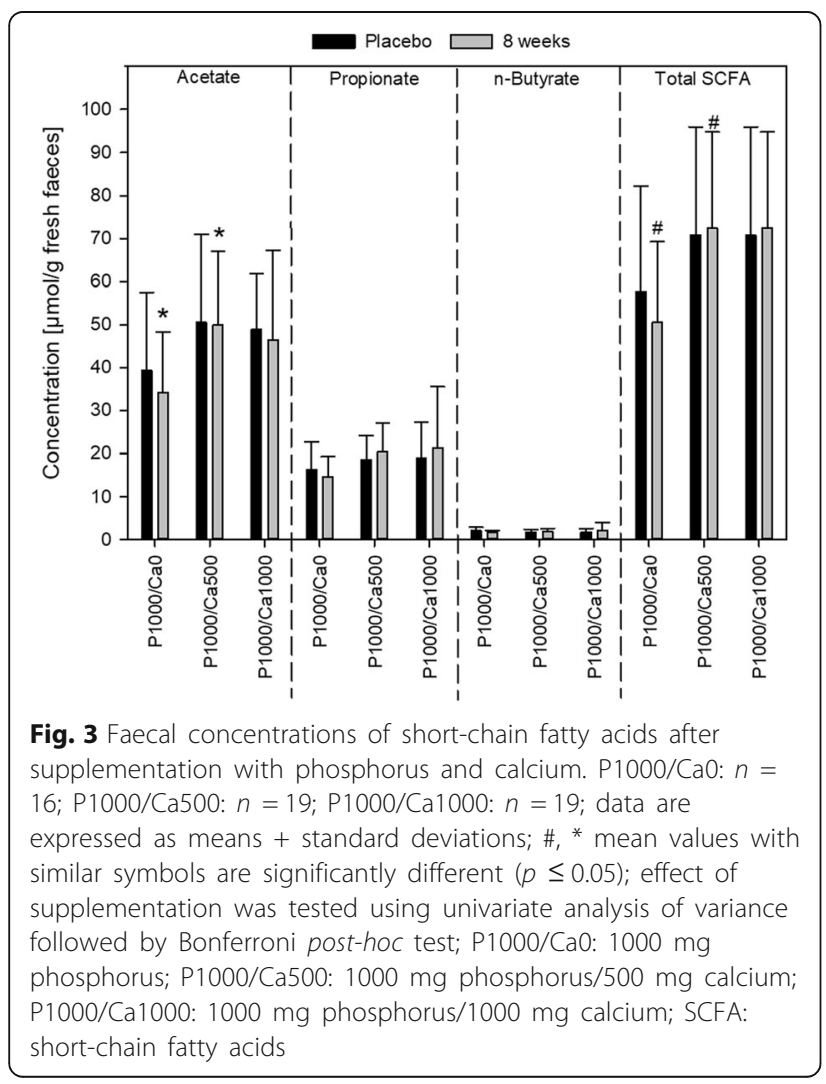

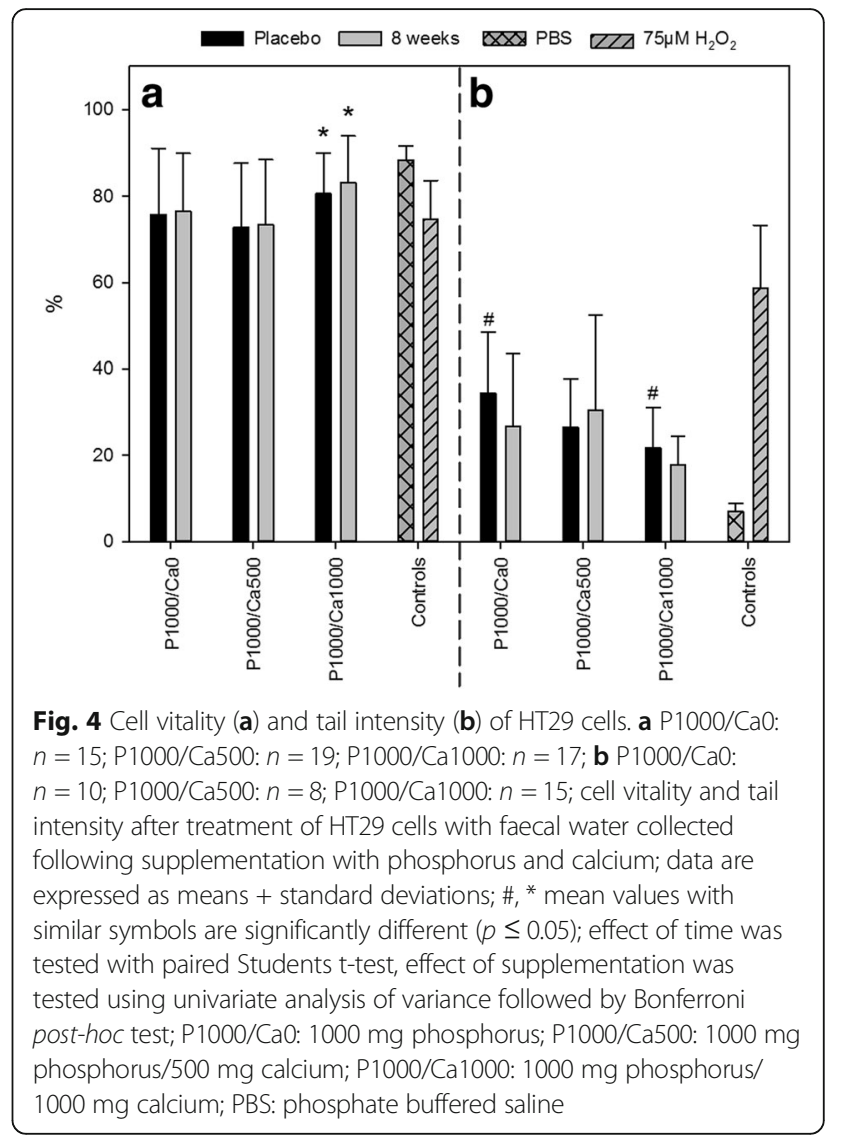

\section{Microbial composition in faeces}

Considering all subjects of the study subgroups $(n=5$ for each intervention), no differences of the microbiome were observed between placebo and eight weeks of intervention as well as between the different intervention groups after eight weeks. Interestingly, men of the P1000/Ca1000 intervention had a significantly different gut microbial community compared to the men of the $\mathrm{P} 1000 / \mathrm{Ca} 0(R=0.778, p=0.01)$ and the $\mathrm{P} 1000 / \mathrm{Ca} 500$ groups $(R=0.593, p=0.01)$ (Fig. 5). Abundance of the genus Acetivibio and of the family Coriobacteriaceae were significantly lower in men after eight weeks of P1000/Ca500 intervention when compared to P1000/ $\mathrm{Ca} 0$ intervention $(p<0.05)$. Clostridium XVIII was significantly more abundant in men of the P1000/Ca1000 intervention group compared to men of the $\mathrm{P} 1000 /$ $\mathrm{Ca} 500(p=0.04)$ and $\mathrm{P} 1000 / \mathrm{Ca} 0(p=0.04)$ groups. The OTUs belonging to Bifidobacterium genus were significantly less abundant in faeces of men of the P1000/ Ca1000 intervention compared to the faeces of men of the P1000/Ca500 group $(p=0.04)$. Figure 6 shows the relative abundance of the OTUs contributing to the group separation of the men per intervention after eight weeks. OTU4, an uncultured bacterium related to Clostridium XVIII, was significantly more abundant in the 


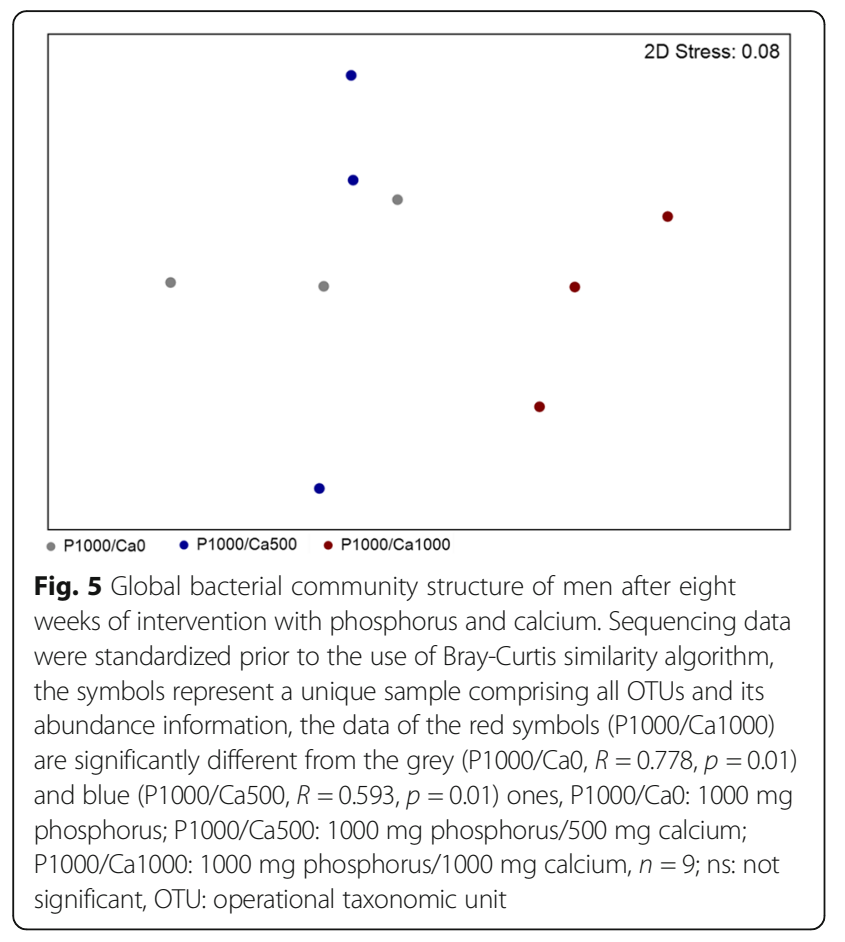

men of P1000/Ca1000 intervention group compared to $\mathrm{P} 1000 / \mathrm{Ca} 500(p=0.03)$ and $\mathrm{P} 1000 / \mathrm{Ca} 0 \quad(p=0.04)$. OTU2, a butyrate-producing bacterium, was significantly more abundant in men of the $\mathrm{P} 1000 / \mathrm{Ca} 0$ intervention group compared to the men with $\mathrm{P} 1000 / \mathrm{Ca} 1000$ supplementation $(p=0.01)$. A trend was observed when the microbial community of men was compared with the one of women $(\mathrm{P} 1000 / \mathrm{Ca} 1000)(p=0.1)$. OTU 4 was more abundant in men while OTU 19 and OTU 28 were more abundant in women $(p<0.03)$ (Additional file 3: Figure S3). Dietary interventions in women did not cause any significant differences of the microbial community.

\section{Discussion}

Already in the 1980s, Newmark et al. hypothesized that calcium ions, bile and fatty acids form insoluble soaps in the human gut, which are detectable in the faeces [29]. Initially, phosphate was considered as a competitor of bile and fatty acids, but Van der Meer et al. showed that phosphate did not compete with bile and fatty acids, but was rather necessary for the

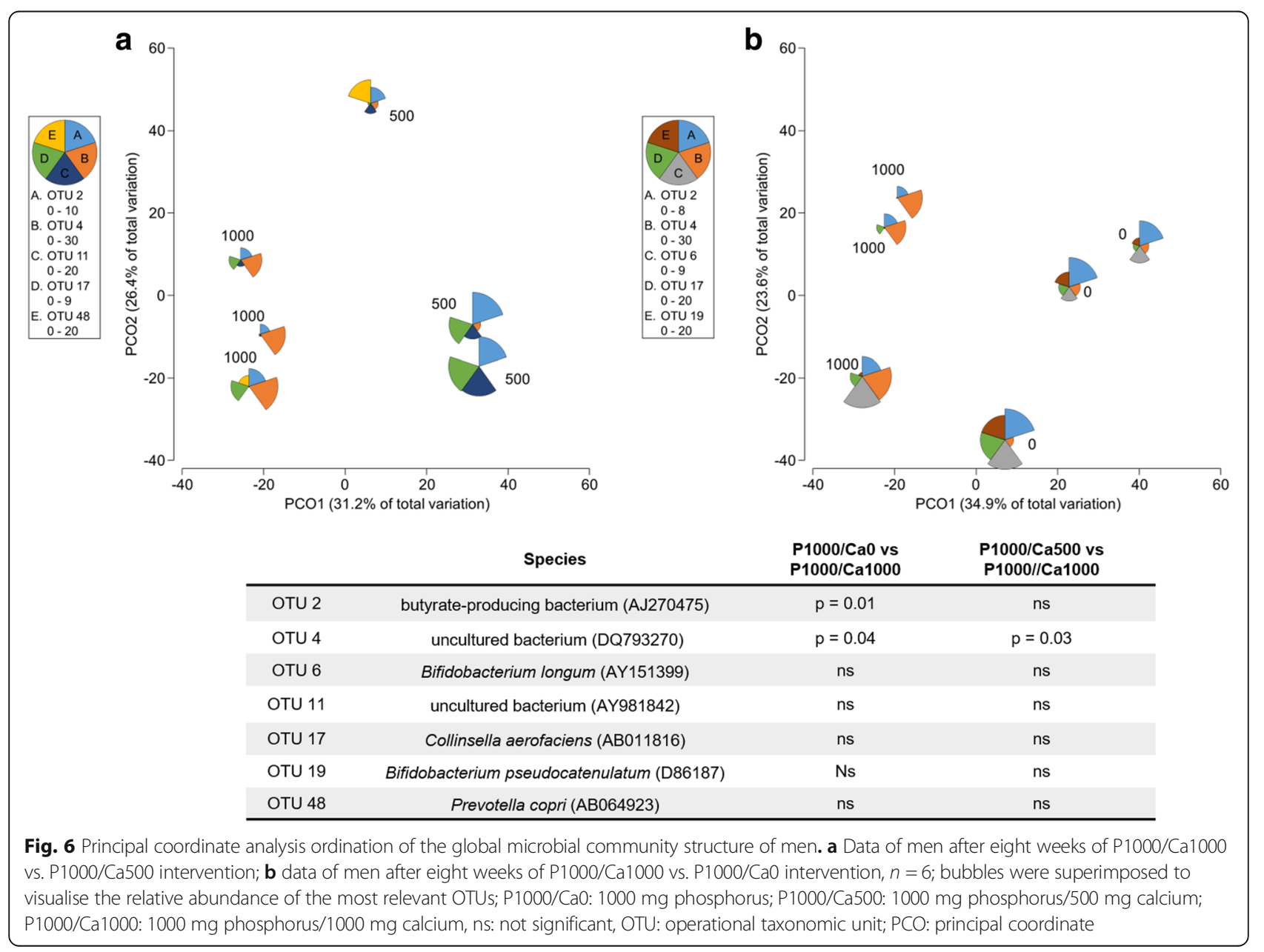


precipitation with calcium [30]. At a molar calciumto-phosphate ratio of $3: 2$ and a $\mathrm{pH}$ value of 5.6 , one part of the ingested dietary calcium and phosphate precipitates as ACP in the small intestine [31]. ACP are able to precipitate both glycine-conjugated and free bile acids by ionic absorption of their negatively charged carboxyl group to the positively charged calcium ions on the surface of the ACP [31]. The hydrophobic tail of the bile acid is exposed to the aqueous phase, which in turn leads to the formation of hydrophobic aggregates. These aggregates are able to bind other hydrophobic ligands [31]. This and the facts that calcium from milk and fermented milk products increases the resistance of rats to salmonella infections [32] and stimulates intestinal lactobacilli colonisation [33], leads to the assumption that dietary calcium and phosphorus can beneficially modify the intestinal environment.

Our human intervention study showed that the faecal concentrations of phosphorus and calcium increased significantly after eight weeks of phosphorus and calcium supplementation [10]. This increase could be due to the formation of ACP. However, even in the group supplemented only with phosphorus indications for ACP formation were observed. We found increasing concentrations of faecal phosphorus without increasing calcium concentrations, but decreasing renal calcium excretion [10]. These results led to the assumption that dietary calcium partly precipitates with phosphate to $\mathrm{ACP}$ and cannot be absorbed in the gut. In order to maintain calcium homeostasis of the body, fewer calcium was excreted via urine.

Formation of ACP is a well-known process in the human gut $[12,13,28]$, and could be a prerequisite for a beneficial modulation of gut health. A decrease of the available intestinal bile acids by precipitation with ACP can lead to a modified microbiota composition and accordingly to a change in SCFA production and composition [34, 35]. The faecal SCFA concentrations of our subjects showed no effect over time (placebo vs. eight weeks of each intervention). But, after eight weeks, the $\mathrm{P} 1000 / \mathrm{Ca} 0$ group showed the lowest concentrations of total SCFA ( $55 \pm 18 \mu \mathrm{mol} / \mathrm{g}$ faeces) compared to P1000/ Ca500 (76 $\pm 22 \mu \mathrm{mol} / \mathrm{g}$ faeces, $p \leq 0.05)$ and $\mathrm{P} 1000 /$ Ca1000 $(73 \pm 31 \mu \mathrm{mol} / \mathrm{g}$ faeces, $p=0.081)$. This higher concentration of total SCFA in the calcium supplemented groups is likely due to a higher acetate concentration. Furthermore, men showed significantly higher total SCFA and acetate concentrations after eight weeks of P1000/Ca1000 intervention compared to the P1000/ $\mathrm{Ca} 0$ one. These effects are independent of the water content of the faeces, since faecal dry matter did not change due to the interventions (data not shown). In a study of Ditscheid, total SCFA $(p=0.065)$ and acetate concentrations ( $p=0.019)$ increased after supplementation with calcium phosphate ( $\beta$-tricalciumphosphate) compared to placebo [36]. The author assumed that either an increased production or a decreased absorption of acetate could be the reason for the higher faecal concentrations after calcium phosphate supplementation. Trinidad et al. showed that bioavailable calcium supports intestinal acetate absorption [37]. Therefore, Ditscheid hypothesized that bioavailability of calcium decreased due to ACP formation and thus acetate absorption is diminished [36]. But, calcium phosphate supplementation has been shown to modulate the intestinal gut community by changing the intestinal environment $[11,33]$. This shift of the gut microbiota could lead to an increased production of SCFA, such as acetate [34].

In the present study, we determined in a subgroup of five subjects per intervention the microbial community in faeces by DNA extraction and amplicon sequencing. Interestingly, men of the $\mathrm{P} 1000 / \mathrm{Ca} 1000$ group showed a shift of the microbial community compared to men of the two other intervention groups. Clostridium XVIII was significantly more abundant in men of the highest calcium group. Furthermore, men of the P1000/Ca1000 intervention group had significantly higher acetate concentrations compared to men of the $\mathrm{P} 1000 / \mathrm{Ca} 0$ one. In general, Clostridium spp. are known to produce acetate by microbial fermentation of carbohydrates in the human gut [34]. Although, the detected Clostridium XVIII is an uncultured bacterium and not characterised so far, it could exhibit similar acetate producing properties. Moreover, OTU2, a butyrate-producing bacterium, was significantly more abundant in men of the P1000/ $\mathrm{Ca} 0$ intervention group and tended to be more abundant in the $\mathrm{P} 1000 / \mathrm{Ca} 500$ group compared to the men of the $\mathrm{P} 1000 / \mathrm{Ca} 1000(p=0.01)$ one. But whether these associations depend on the calcium and phosphorus supplementation remains unclear, since we were only able to study the microbial community of a subgroup of five subjects per intervention. The partly gender-specific effects might be a result of sex-specific diet effects on the human microbiome as proposed by Bolnick et al. [38]. Animal and human studies showed, that gender influences microbial composition in the gut [38-41] and, thus, the formation of SCFA [42], too.

A modulation of the cyto- and genotoxicity of the FW after calcium and phosphorus supplementation is discussed since ACP are known to precipitate secondary bile acids in the gut $[29,43]$. Secondary bile acids showed cyto- and genotoxic effects on human normal and tumour colon cells [44-46]. Our results indicate that the phosphorus supplementation with and without calcium did not affect the genotoxicity of FW and did not markedly effect the vitality of HT29 cells. Similar results were provided by Ditscheid et al., which could 
not show changes in the genotoxicity of FW after calcium phosphate supplementation [47]. Glinghammer et al. reported that cytotoxicity of FW increased after subjects switched from a dairy product-rich (high calcium) to a dairy product-free diet (lower calcium), but genotoxicity did not alter [48]. The authors suggested that the mechanism by which dairy products may act colon cancer protective are at the level of tumour promotion and indicated that dairy products, especially calcium and phosphate, exert protective effects by precipitating bile and fatty acids [48]. Gomes et al. summarised in a review that high calcium diets reduce FW cytotoxicity by precipitating cytotoxic surfactants, which results in a lower colonic epithelium damage and higher resistance against infections [49]. All studies cited in this context used a calcium phosphate supplement, but almost all are based on animal models. Furthermore, the cell model systems and methods used were different to the ones used here, e.g. cytotoxicity was tested with erythrocytes [43] instead of intestinal cells. Besides, maybe a more realistic picture of the FW genotoxicity would be displayed by a quantitative stool sampling over one or more days. Unfortunately, parameters in faeces were not the main outcome of our human intervention study and so only one stool sample was collected.

Like the precipitation of bile acids, ACP are in principle able to precipitate fatty acids as well [49]. The resulting increased faecal excretion of fat could favour weight control [50]. Our study did not show changes in faecal fat concentrations in response to the phosphorus and calcium supplementation. Ditscheid et al. also reported no significant changes in faecal fat excretion after supplementation with $\beta$-tricalciumphosphate compared to a normal calcium intake (approx. 2200 vs. $1200 \mathrm{mg}$ calcium/d and 2000 vs. $1600 \mathrm{mg}$ phosphorus/ d) [13]. In contrast, Boon et al. showed a non-significant $57 \%$ increase in faecal fat after high compared to low calcium intakes (2500 vs $400 \mathrm{mg}$ calcium/d, low-fat dairy) [51]. Furthermore, in a study by Bendsen et al. faecal fat increased from 5.4 to $11.5 \mathrm{~g} / \mathrm{d}$ after intake of $1600 \mathrm{mg}$ calcium/d (by dairy supplementation) [52]. Another study comparing low (approx. $500 \mathrm{mg}$ calcium/ d) with high calcium diets (approx. $1800 \mathrm{mg}$ calcium/d, low-fat dairy products) showed a 2.5 -fold increase in faecal fat after the high calcium intake [53]. One reason for the discrepancies could be the standardising of the diet and stool collection time. In the study presented here a dietary record for three days was done and one faecal sample was collected. However, the study of Ditscheid et al. included a standardised diet and a five-day faecal collection. But, nevertheless they also found no effect on faecal fat concentration. Possibly, this could be caused by the normal calcium intake that was used as placebo here (approx. $900 \mathrm{mg}$ calcium/d) [10] and by Ditscheid et al. (approx. $1200 \mathrm{mg}$ calcium/d) [13], whereas in the studies by Boon et al. (400 $\mathrm{mg}$ calcium/d) [51] and Jacobsen et al. (500 mg calcium/d) [53] low calcium diets were used as controls. It is therefore possible that the observed changes in faecal fat concentrations were caused by decreasing the intake of calcium in the controls to levels below the norm.

In a phosphate supplementation study of Grimm et al., a phosphate-rich diet (additional $1436 \mathrm{mg}$ phosphorus/ d) was associated with intestinal distress, soft stool or mild diarrhoea. The authors concluded that the symptoms are a consequence of an osmotic effect of the added polyphosphates in the intestinal lumen [15]. In our study $20-40 \%$ of the subjects in each intervention group and at each time point reported intestinal disturbances. The majority of the subjects mentioned a connection to the test product. Analysis of the questionnaires showed that the reported disturbances were independent of the phosphorus and calcium supplementation and time-point of intervention. Therefore, we consider the gut problems as a result of the sherbet powder consumption in general and not as a result of the phosphorus and calcium supplementation. Another reason could be the focus of the subjects on the new diet component (sherbet powder) and a resulting overestimation of intestinal distress in case of gut problems. Furthermore, the interpretation of the questionnaires is limited, since the subjects reported only whether they had problems but not the frequency and intensity of disturbances.

The most important limitations of our study are (i) the restricted one portion faecal collection and (ii) the determination of gut community in a limited subgroup with only a few subjects.

To the best of our knowledge, this is the first human intervention study in the field of high phosphorus and calcium intakes, which also paid attention on gut health. As the main part of the additional phosphorus and calcium passes the whole gut via ACP and seems to modulate gut physiology, further human intervention studies in this context are needed.

\section{Conclusions}

In conclusion, the high phosphorus supplementation did not influence cyto- and genotoxicity of FW as well as the concentrations of faecal fat independent of calcium intake. Our study provides first hints for a potential phosphorus-induced modulation of the gut community and the faecal total SCFA content. Since additional dietary phosphorus and calcium passes the gut as ACP, further studies are needed to evaluate the effect of high phosphorus and calcium diets on gut-related parameters. 


\section{Additional files}

Additional file 1: Figure S1. Faecal concentrations of short-chain fatty acids after supplementation with phosphorus and calcium of men. P1000/Ca0: $n=7 ;$ P1000/Ca500: $n=9 ;$ P1000/Ca1000: $n=8$; data are expressed as means + standard deviations; $\#,{ }^{*}$ mean values with similar symbols are significant different ( $p \leq 0.05$ ); effect of supplementation was tested using univariate analysis of variance followed by Bonferroni posthoc test; a, b significantly different (Wilcoxon sign-rank test); P1000/Ca0: 1000 mg phosphorus; P1000/Ca500: 1000 mg phosphorus/500 mg calcium; P1000/Ca1000: 1000 mg phosphorus/1000 mg calcium; SCFA: short-chain fatty acids. (PNG $36 \mathrm{~kb}$ )

Additional file 2: Figure S2. Faecal concentrations of short-chain fatty acids after supplementation with phosphorus and calcium of women. P1000/Ca0: $n=9 ;$ P1000/Ca500: $n=10 ;$ P1000/Ca1000: $n=11$; data are expressed as means + standard deviations; \#, ${ }^{*}$ mean values with similar symbols are significant different ( $p \leq 0.05$ ); effect of time was tested with paired Students t-test; effect of supplementation was tested using univariate analysis of variance followed by Bonferroni post-hoc test; P1000/Ca0: 1000 mg phosphorus; P1000/Ca500: 1000 mg phosphorus/ 500 mg calcium; P1000/Ca1000: 1000 mg phosphorus/1000 mg calcium; SCFA: short-chain fatty acids. (PNG $35 \mathrm{~kb}$ )

Additional file 3: Figure S3. Principal coordinate analysis ordination of the global community structure of men and women after eight weeks of P1000/Ca1000 intervention. $n=5$, bubbles were superimposed to visualise the relative abundance of the most relevant OTUs; P1000/ Ca1000: 1000 mg phosphorus/1000 mg calcium, ns: not significant, OTU: operational taxonomic unit; PCO: principal coordinate. (TIFF 576 kb)

\section{Abbreviations}

ACP: Amorphous calcium phosphate; FW: Faecal water; OTU: Operational taxonomic unit; P1000/Ca0: 1000 mg phosphorus; P1000/Ca1000: 1000 mg phosphorus/1000 mg calcium; P1000/Ca500: 1000 mg phosphorus/500 mg calcium; PBS: Phosphate buffered saline; PCO: Principal coordinate; SCFA: Short-chain fatty acids; TI: Tail intensity

\section{Acknowledgements}

Technical assistance is greatly appreciated from Ute Helms, Esther Woschee, Kerstin Kalmring-Raspe und Carsten Rohrer.

\section{Funding}

Phosphoric Acid \& Phosphate Producers Associations (PAPA) funded the project. PAPA was not involved in the study design and did not influence the interpretation of the results.

\section{Availability of data and materials}

The datasets used and/or analysed during the current study are available from the corresponding author on reasonable request.

\section{Authors' contributions}

All authors made substantial contributions to one or more of the following: study conception and design, acquisition of data, analysis and interpretation of data, drafting and/or critically revising the manuscript for important intellectual content. Each author has seen and approved the contents of the submitted manuscript. None of the authors had any personal or financial conflicts of interests. UT conducted research, analysed data, performed statistical analysis and wrote the manuscript; ACS extracted samples and performed statistical and bioinformatics analysis of sequencing data; SL analysed SCFA data; UT, GJ and MG designed research and had primary responsibility for final content. All authors were responsible for the critical revision of the manuscript. All authors read and approved the final manuscript.

\section{Ethics approval and consent to participate}

This study was approved by the Ethical Committee of the Friedrich Schiller University Jena (no: 3987-01/14). Written informed consent was obtained from all subjects. The trial is registered at ClinicalTrials.gov as NCT02095392.
Consent for publication

Not applicable

\section{Competing interests}

The authors declare that they have no competing interests.

\section{Publisher's Note}

Springer Nature remains neutral with regard to jurisdictional claims in published maps and institutional affiliations.

\section{Author details}

${ }^{1}$ Department of Nutritional Toxicology, Institute of Nutrition, Friedrich Schiller University Jena, Dornburger Straße 24, 07743 Jena, Germany. ${ }^{2}$ Institute of Animal Science, University of Hohenheim, Emil-Wolff-Straße. 10, 70599 Stuttgart, Germany. ${ }^{3}$ Department of Nutritional Physiology, Institute of Nutrition, Friedrich Schiller University Jena, Dornburger Straße 23, 07743 Jena, Germany. ${ }^{4}$ Department of Nutritional Biochemistry and Physiology, Institute of Nutrition, Friedrich Schiller University Jena, Dornburger Straße 25, 07743 Jena, Germany. ${ }^{5}$ Competence Cluster for Nutrition and Cardiovascular Health (nutriCARD) Halle-Jena-Leipzig, Jena, Germany.

Received: 21 August 2017 Accepted: 22 January 2018

Published online: 16 February 2018

\section{References}

1. Calvo MS, Uribarri J. Public health impact of dietary phosphorus excess on bone and cardiovascular health in the general population. Am J Clin Nutr. 2013:98:6-15.

2. Dhingra R, Sullivan LM, Fox CS, Wang TJ, D'Agostino RB, Gaziano JM, Vasan RS. Relations of serum phosphorus and calcium levels to the incidence of cardiovascular disease in the community. Arch Intern Med. 2007;167:879-85.

3. Eddington $\mathrm{H}$, Hoefield R, Sinha S, Chrysochou C, Lane B, Foley RN, Hegarty J, New J, O'Donoghue DJ, Middleton RJ, Kalra PA. Serum phosphate and mortality in patients with chronic kidney disease. Clin J Am Soc Nephrol. 2010;5:2251-7.

4. Chang AR, Lazo M, Appel L, Gutierrez OM, Grams ME. High dietary phosphorus intake is associated with all-cause mortality: results from NHANES III. Am J Clin Nutr. 2014;99:320-7.

5. de Boer $\mathbb{H}$, Rue TC, Kestenbaum B. Serum phosphorus concentrations in the third National Health and Nutrition Examination Survey (NHANES III). Am J Kidney Dis. 2009;53:399-407.

6. Selamet U, Tighiouart H, Sarnak MJ, Beck G, Levey AS, Block G, Ix JH. Relationship of dietary phosphate intake with risk of end-stage renal disease and mortality in chronic kidney disease stages 3-5: the modification of diet in renal disease study. Kidney Int. 2016;89:176-84.

7. Adatorwovor R, Roggenkamp K, Anderson JJ. Intakes of calcium and phosphorus and calculated calcium-to-phosphorus ratios of older adults: NHANES 2005-2006 data. Nutrients. 2015;7:9633-9.

8. Calvo MS, Uribarri J. Contributions to total phosphorus intake: all sources considered. Semin Dial. 2013;26:54-61.

9. Ritz E, Hahn K, Ketteler M, Kuhlmann MK, Mann J. Phosphate additives in food-a health risk. Dtsch Arztebl Int. 2012;109:49-55.

10. Trautvetter $\mathrm{U}$, Jahreis $\mathrm{G}$, Kiehntopf $\mathrm{M}$, Glei M. Consequences of a high phosphorus intake on mineral metabolism and bone remodeling in dependence of calcium intake in healthy subjects - a randomized placebocontrolled human intervention study. Nutr J. 2016;15:7.

11. Trautvetter $U$, Ditscheid B, Kiehntopf M, Jahreis G. A combination of calcium phosphate and probiotics beneficially influences intestinal lactobacilli and cholesterol metabolism in humans. Clin Nutr. 2012;31:230-7.

12. Trautvetter U, Neef N, Leiterer M, Kiehntopf M, Kratzsch J, Jahreis G. Effect of calcium phosphate and vitamin D3 supplementation on bone remodelling and metabolism of calcium, phosphorus, magnesium and iron. Nutr J. 2014;13:6.

13. Ditscheid B, Keller $\mathrm{S}$, Jahreis $\mathrm{G}$. Cholesterol metabolism is affected by calcium phosphate supplementation in humans. J Nutr. 2005;135:1678-82.

14. Van der Meer R, Lapre JA, Govers M, Kleibeuker JH. Mechanisms of the intestinal effects of dietary fats and milk products on colon carcinogenesis. Cancer Lett. 1997;114:75-83.

15. Grimm M, Müller A, Hein G, Fünfstück R, Jahreis G. High phosphorus intake only slightly affects serum minerals, urinary pyridinium crosslinks and renal function in young women. Eur J Clin Nutr. 2001;55:153-61. 
16. Matsushita K, Tonelli M, Lloyd A, Levey AS, Coresh J, Hemmelgarn BR. Clinical risk implications of the CKD Epidemiology Collaboration (CKD-EPI) equation compared with the Modification of Diet in Renal Disease (MDRD) study equation for estimated GFR. Am J Kidney Dis. 2012;60:241-9.

17. Roessler A, Forssten SD, Glei M, Ouwehand AC, Jahreis G. The effect of probiotics on faecal microbiota and genotoxic activity of faecal water in patients with atopic dermatitis: a randomized, placebo-controlled study. Clin Nutr. 2012;31:22-9.

18. Klinder A, Karlsson PC, Clune Y, Hughes R, Glei M, Rafter JJ, Rowland I, Collins JK, Pool-Zobel BL. Fecal water as a non-invasive biomarker in nutritional intervention: comparison of preparation methods and refinement of different endpoints. Nutr Cancer. 2007;57:158-67.

19. Glei M, Kirmse A, Habermann N, Persin C, Pool-Zobel BL. Bread enriched with green coffee extract has chemoprotective and antigenotoxic activities in human cells. Nutr Cancer. 2006;56:182-92.

20. Munjal U, Scharlau D, Glei M. Gut fermentation products of inulin-type fructans modulate the expression of xenobiotic-metabolising enzymes in human colonic tumour cells. Anticancer Res. 2012;32:5379-86.

21. Knoll N, Weise A, Claussen U, Sendt W, Marian B, Glei M, Pool-Zobel BL. 2Dodecylcyclobutanone, a radiolytic product of palmitic acid, is genotoxic in primary human colon cells and in cells from preneoplastic lesions. Mutat Res. 2006:594:10-9.

22. Oberreuther-Moschner DL, Jahreis G, Rechkemmer G, Pool-Zobel BL. Dietary intervention with the probiotics lactobacillus acidophilus 145 and Bifidobacterium longum 913 modulates the potential of human faecal water to induce damage in HT29clone19A cells. Br J Nutr. 2004;91:925-32.

23. Glei M, Matuschek M, Steiner C, Böhm V, Persin C, Pool-Zobel BL. Initial in vitro toxicity testing of functional foods rich in catechins and anthocyanins in human cells. Toxicol in Vitro. 2003;17:723-9.

24. Camarinha-Silva A, Jauregui R, Chaves-Moreno D, Oxley AP, Schaumburg F Becker K, Wos-Oxley ML, Pieper DH. Comparing the anterior nare bacterial community of two discrete human populations using Illumina amplicon sequencing. Environ Microbiol. 2014;16:2939-52.

25. Kozich JJ, Westcott SL, Baxter NT, Highlander SK, Schloss PD. Development of a dual-index sequencing strategy and curation pipeline for analyzing amplicon sequence data on the MiSeq Illumina sequencing platform. Appl Environ Microbiol. 2013;79:5112-20.

26. Wang Q, Garrity GM, Tiedje JM, Cole JR. Naive Bayesian classifier for rapid assignment of rRNA sequences into the new bacterial taxonomy. Appl Environ Microbiol. 2007;73:5261-7.

27. Clarke KR, Warwick RM: Change in Marine Communities: an approach to statistical analysis and Interpretation. Plymouth marine laboratory, Natural environment research council; 1994.

28. Welch BL. The generalization of 'Student's' problem when several different population variances are involved. Biometrika. 1947;34:28-35.

29. Newmark HL, Wargovich MJ, Bruce WR. Colon cancer and dietary fat, phosphate, and calcium: a hypothesis. J Natl Cancer Inst. 1984;72:1323-5.

30. Van der Meer R, Termont DS, De Vries HT. Differential effects of calcium ions and calcium phosphate on cytotoxicity of bile acids. Am J Phys. 1991;260:G142-7.

31. Govers MJAP, Termont DSML, Van der Meer R. Mechanism of the antiproliferative effect of milk mineral and other calcium supplements on colonic epithelium. Cancer Res. 1994;54:95-100.

32. Bovee-Oudenhoven I, Termont D, Dekker R, Van der Meer R. Calcium in milk and fermentation by yoghurt bacteria increase the resistance of rats to salmonella infection. Gut. 1996:38:59-65.

33. Bovee-Oudenhoven IM, Wissink ML, Wouters JT, Van der Meer R. Dietary calcium phosphate stimulates intestinal lactobacilli and decreases the severity of a salmonella infection in rats. J Nutr. 1999;129:607-12.

34. Koh A, De Vadder F, Kovatcheva-Datchary P, Bäckhed F. From dietary fiber to host physiology: short-chain fatty acids as key bacterial metabolites. Cell. 2016;165:1332-45.

35. Bovee-Oudenhoven IM, Termont DS, Weerkamp AH, Faassen-Peters MA, Van der Meer R. Dietary calcium inhibits the intestinal colonization and translocation of salmonella in rats. Gastroenterology. 1997;113:550-7.

36. Ditscheid B: In vivo investigation into the physiologic effects of pentacalcium hydroxy-triphosphate supplementation in humans and in vitro model for the adsorption of bile acids and neutral sterols to different calcium phosphates. Dissertation; 2006.

37. Trinidad TP, Wolever TM, Thompson LU. Effects of calcium concentration, acetate, and propionate on calcium absorption in the human distal colon. Nutrition. 1999;15:529-33.
38. Bolnick DI, Snowberg LK, Hirsch PE, Lauber CL, Org E, Parks B, Lusis AJ, Knight R, Caporaso JG, Svanbäck R. Individual diet has sex-dependent effects on vertebrate gut microbiota. Nat Commun. 2014;5:4500.

39. Org E, Mehrabian M, Parks BW, Shipkova P, Liu X, Drake TA, Lusis AJ. Sex differences and hormonal effects on gut microbiota composition in mice. Gut Microbes. 2016;7:313-22.

40. Neuman H, Debelius JW, Knight R, Koren O. Microbial endocrinology: the interplay between the microbiota and the endocrine system. FEMS Microbiol Rev. 2015;39:509-21.

41. Most J, Goossens GH, Reijnders D, Canfora EE, Penders J, Blaak EE. Gut microbiota composition strongly correlates to peripheral insulin sensitivity in obese men but not in women. Benefic Microbes. 2017:8:557-62.

42. Wong JMWRD, de Souza RRD, Kendall CWCP, Emam AM, Jenkins DJAMD. Colonic health: fermentation and short chain fatty acids. J Clin Gastroenterol. 2006:40:235-43.

43. Govers MJ, Termont DS, Lapre JA, Kleibeuker JH, Vonk RJ, Van der Meer R. Calcium in milk products precipitates intestinal fatty acids and secondary bile acids and thus inhibits colonic cytotoxicity in humans. Cancer Res. 1996;56:3270-5.

44. Rosignoli P, Fabiani R, De Bartolomeo A, Fuccelli R, Pelli MA, Morozzi G. Genotoxic effect of bile acids on human normal and tumour colon cells and protection by dietary antioxidants and butyrate. Eur J Nutr. 2008;47: 301-9.

45. Tong JL, Ran ZH, Shen J, Fan GQ, Xiao SD. Association between fecal bile acids and colorectal cancer: a meta-analysis of observational studies. Yonsei Med J. 2008:49:792-803.

46. Powolny A, Xu J, Loo G. Deoxycholate induces DNA damage and apoptosis in human colon epithelial cells expressing either mutant or wild-type p53. Int J Biochem Cell Biol. 2001;33:193-203.

47. Ditscheid B, Keller $\mathrm{S}$, Jahreis $\mathrm{G}$. Faecal steroid excretion in humans is affected by calcium supplementation and shows gender-specific differences. Eur J Nutr. 2009;48:22-30.

48. Glinghammar B, Venturi M, Rowland IR, Rafter JJ. Shift from a dairy productrich to a dairy product-free diet: influence on cytotoxicity and genotoxicity of fecal water-potential risk factors for colon cancer. Am J Clin Nutr. 1997; 66:1277-82

49. Gomes JM, Costa JA, Alfenas RC. Could the beneficial effects of dietary calcium on obesity and diabetes control be mediated by changes in intestinal microbiota and integrity? Br J Nutr. 2015;114:1756-65.

50. Christensen R, Lorenzen JK, Svith CR, Bartels EM, Melanson EL, Saris WH, Tremblay A, Astrup A. Effect of calcium from dairy and dietary supplements on faecal fat excretion: a meta-analysis of randomized controlled trials. Obes Rev. 2009;10:475-86.

51. Boon N, Hul GBJ, Stegen JHCH, Sluijsmans WEM, Valle C, Langin D, Viguerie $\mathrm{N}$, Saris WHM. An intervention study of the effects of calcium intake on faecal fat excretion, energy metabolism and adipose tissue mRNA expression of lipid-metabolism related proteins. Int J Obes. 2007;31:1704-12.

52. Bendsen NT, Hother AL, Jensen SK, Lorenzen JK, Astrup A. Effect of dairy calcium on fecal fat excretion: a randomized crossover trial. Int J Obes. 2008; 32:1816-24.

53. Jacobsen R, Lorenzen JK, Toubro S, Krog-Mikkelsen I, Astrup A. Effect of short-term high dietary calcium intake on 24-h energy expenditure, fat oxidation, and fecal fat excretion. Int J Obes. 2005;29:292-301.

\section{Submit your next manuscript to BioMed Central and we will help you at every step:}

- We accept pre-submission inquiries

- Our selector tool helps you to find the most relevant journal

- We provide round the clock customer support

- Convenient online submission

- Thorough peer review

- Inclusion in PubMed and all major indexing services

- Maximum visibility for your research

Submit your manuscript at www.biomedcentral.com/submit 